\title{
Vincristine treatment revealing asymptomatic hereditary motor sensory neuropathy type $1 \mathrm{~A}$
}

\author{
E Mercuri, J Poulton, J Buck, V Broadbent, M Bamford, H Jungbluth, A Y Manzur, \\ F Muntoni
}

\begin{abstract}
A 5 year old boy developed severe weakness after receiving vincristine for treatment of acute lymphoblastic leukaemia. Although weakness improved after the discontinuation of vincristine, other symptoms suggestive of a neuropathy persisted. Neurophysiological and genetic analysis at age 8 years indicated that vincristine had induced symptoms of a hereditary sensory motor neuropathy type $1 \mathrm{~A}$, which had previously been asymptomatic; his genetically affected mother was also asymptomatic.

(Arch Dis Child 1999;81:442-443)
\end{abstract}

Keywords: vincristine; hereditary sensory motor neuropathy type $1 \mathrm{~A}$; neuropathy; acute lymphoblastic leukaemia

Acute neuropathy is a recognised side effect of treatment with vincristine in patients with leukaemia or other tumours. The neuropathy usually results in an axonal injury and relative preservation of the myelin sheet with normal or only mildly delayed nerve conduction velocity.

Department of Paediatrics \& Neonatal Medicine, Imperial College School of Medicine,

Hammersmith

Hospital, Du Cane

Road, London

W12 0HN, UK

E Mercuri

$\mathrm{H}$ Jungbluth

AY Manzur

F Muntoni

Department of Genetics, Radcliffe

Hospital, Oxford OX9 3DU, UK

J Poulton

Department of Paediatric Oncology, Addenbrooke's Hospital, Hills Road, Cambridge CB2 2QQ, UK

J Buck

V Broadbent

Ipswich Hospital, Heath Road, Ipswich IP4 5PD, UK

M Bamford

Correspondence to: Dr Mercuri

email: e.mercuri@ic.ac.uk

Accepted 13 July 1999 falling over and complained of aching legs. A few days later he developed fever, lethargy, rash, and was unable to stand and walk. A diagnosis of acute lymphoblastic leukaemia (ALL) was made. The child partially recovered and started walking again but he had an abnormal gait and difficulties in rising from the floor. $\mathrm{He}$ was started on vincristine (induction dose $1.5 \mathrm{mg} / \mathrm{m}^{2}$ ) as suggested by the UK ALL XI protocol, and during the following weeks his weakness progressed very rapidly to the point that he lost ambulation and the ability to sit up. He only had partial subgravity movements of the legs and arms - for example, he was unable to raise his arm fully against gravity. Distal weakness was also noticed, in particular he had difficulties extending his fingers. He subsequently developed bulbar signs with speech and swallowing difficulties and had to be admitted to the intensive care unit where he remained for 10 days. His reflexes disappeared. Vincristine was discontinued four weeks after the first dose was given and his symptoms improved. Three months after stopping vincristine he was able to stand and walk with support, and had no residual bulbar weakness. The recovery continued through his second course three months later. One year after diagnosis he had further improved and was able to walk without support. He had been cured from his leukaemia. The child was referred to us for a second opinion 2.5 years after the diagnosis of the leukaemia. On examination he was able to walk with a foot drop, to jump, and almost to hop. He was able to rise from the floor without a Gowers' manoeuvre. Cranial nerves were normal. Muscle power was slightly reduced in the trunk and proximally, and more reduced distally, especially in the ankle dorxiflexion bilaterally, where it was subgravity. Sensation was normal, reflexes were absent. He still had some mild difficulties in fine motor abilities and handwriting.

The muscle ultrasound was normal. Nerve conduction velocity was reduced in both ulnar and peroneal nerves $(22.7$ and $28.3 \mathrm{~m} / \mathrm{s}$, respectively). The amplitude was also low $(<50 \mu \mathrm{V})$. These finding were suggestive of a demyelinating neuropathy.

Although the family history was negative, because demyelinating peripheral neuropathies are often inherited in a dominant way, we assessed nerve conduction in the boy's mother; this showed a slowing of conduction in both median and peroneal nerves $(25$ and $27.3 \mathrm{~m} / \mathrm{s}$, respectively). In both the boy and his mother, DNA analysis showed a duplication at 17p11.2 suggesting a diagnosis of HSMN type $1 \mathrm{~A}$.
13 months, and was able to join his peers in the playground without difficulty. The child had been well until the age of 5 when he started 


\section{Discussion}

This case suggests that vincristine treatment had induced weakness in a child with an otherwise asymptomatic hereditary sensory motor neuropathy. Despite the absence of symptoms before treatment or positive family history, the severity and the persistence of the symptoms two years after the discontinuation of vincristine suggested an underlying additional cause. Neurophysiological findings and genetic analysis confirmed HMSN type 1A in the child. Surprisingly, these investigations suggested that his asymptomatic mother is similarly affected by HMSN type $1 \mathrm{~A}$.

These findings suggest that a peripheral motor sensory neuropathy has to be suspected in cases of unusual severity of vincristine neuropathy even when the clinical and family histories are negative. They also suggest that, when considering using vincristine, either chromosome $17 \mathrm{p} 11.2$ duplication studies or nerve conduction velocity should be performed to rule out an underlying HSMN type 1. This will help to identify patients at risk of developing severe signs of neuropathy following vincristine induction, in whom treatment with other drugs, such as vinblastine, should be considered.

1 Casey EB, Jellife AM, Le Quesne PM, Millett YL. Vincristine neuropathy: clinical and electrophysiological observations. Brain 1973;96:69-86.

2 McGuire SA, Ghospe SM, Dahl G. Acute vincristine neurotoxicity in the presence of hereditary motor and sensory neuropathy type I. Med Pediatr Oncol 1989;17:520-3.

3 Graf WD, Chance PF, Lensch MW, Eng LJ, Lipe HP, Bird TD. Severe vincristine neuropathy in Charcot-MarieTooth disease type 1A. Cancer 1996;177:1356-62.

4 Igarashi M, Thompson EI, Rivera GK Vincristine neuropathy in type I and type II Charcot-Marie-Tooth disease (hereditary motor sensory neuropathy). Med Pediatr Oncol 1995;25:113-16.

5 Geny C, Gaio JM, Mallaret M, et al. Maladie de Charcot-Marie-Tooth revelee par un traitment a la vincristine au course d'une maladie de Hodgkin familiale. Ann Med Interne (Paris) 1990;141:709-10.

6 Neumann Y, Toren A, Rechavi G, et al. Vincristine treatment triggering the expression of asymptomatic Charcot Marie Tooth Disease. Med Pediatr Oncol 1996;26:280-3.

\section{FETAL AND NEONATAL EDITION}

\section{November issue}

The following articles - being published in the November 1999 issue of the Fetal and Neonatal edition of the Archives of Disease in Childhood - may be of general interest to paediatricians.

Echocardiographic flow pattern of patent ductus arteriosus: a guide to indomethacin treatment in premature infants

Bai-Horng Su, Ching-Tien Peng, Chang-Hai Tsai

Prone and left lateral positioning reduce gastro-oesophageal reflux in preterm infants

A K Ewer, $M$ E Fames, $\mathcal{F} M$ Tobin

Effect of maternal anticonvulsant treatment on neonatal blood coagulation

Edmund Hey

\section{ETHICS}

Children of the '90s II: challenges for the ethics and law committee $S$ E Mumford 